\title{
Retroperitoneal Sarcoma Involving Unilateral Double Ureter: Management, Treatment and Psychological Implications
}

\author{
Vito Leanza Claudia Garraffo Gianluca Leanza Antonio Leanza \\ Surgery Department, Obstetric and Gynaecologic Unit, Santo Bambino Hospital, \\ Catania University, Catania, Italy
}

\section{Key Words}

Retroperitoneal sarcoma · Double ureters · Quality of life · Psychological implications

\begin{abstract}
The case of a 45-year-old woman who was admitted to our university hospital for polymenorrhea, weight gain and pain in the left iliac region is reported. An abdominal ultrasound revealed a $9.5 \times 5.2-\mathrm{cm}$, hypoechoic and inhomogeneous mass located on the left side of the pelvis and behind the ovary. The patient underwent surgery. The pelvic mass was firmly anchored to the small intestine, colon, sigma and uterine fundus. After removing the adhesions, double ureters, which had been incorporated in the mass, were observed on the left side. Resection of the unilateral double ureters was necessary in order to remove the entire mass, and thereafter, a left salpingoophorectomy was performed. A histological examination showed a malignant retroperitoneal mass. Termino-terminal ureteral anastomosis with two double-J stents was carried out. Total hysterectomy with preservation of the right adenexum and regional lymphadenectomy was performed. The purpose of this case report is to discuss the physical and psychological implications related to the combination of two rare entities: leiomyosarcoma and a double ureter located within the mass. A literature review on the clinical management and psychological aspects from a female cancer patient's perspective undergoing surgery with the aforementioned disorders will be discussed.
\end{abstract}


Leanza et al.: Retroperitoneal Sarcoma Involving Unilateral Double Ureter: Management, Treatment and Psychological Implications

\section{Introduction}

Soft tissue retroperitoneal sarcoma (RPS) is an unusual entity. It accounts for $12-15 \%$ of all sarcomas and $0.2-0.5 \%$ of all tumors [1]. When the mass develops inside the retroperitoneal space, RPS has a propensity to remain asymptomatic for a long period and can consequently grow quite large before diagnosis. RPS, characterized by an increased size and inhomogeneity, is often suspicious for malignancy, and locoregional involvement is common. In addition, RPS is often associated with psychological and social problems such as anxiety, depression, distress, fear of recurrence, lack of social support/function, relationships as well as the impact on family and quality of life (QoL).

The primary intervention for a soft tissue sarcoma is removal. Consequently, surgery is a main part of treatment whenever possible. The operation must be thorough and a suitable expertise is required. A good operation is the key to recovery. However, one must not forget the risk of metastasis. In many cases, for this reason, a polyspecialistic team is required.

\section{Case Presentation}

A 45-year-old woman was admitted to our university hospital for polymenorrhea, weight gain and pain in the left iliac region. Before admission, a Pap smear and a diagnostic hysteroscopy were carried out; the former tested negative, while the latter showed simple endometrial hyperplasia. During the physical examination, the patient was in a good condition and without weight loss. An abdominal examination revealed a flat and painless abdomen, and no palpable mass was found. During the gynecological visit, the uterus was larger in size, while the right ovary and fallopian tube were within expected limits. On the left side, an orange-shaped mass was observed; it had irregular layers, was fixed and painless. An abdominal ultrasound revealed a hypoechoic, inhomogeneous, $9.5 \times 5.2-\mathrm{cm}$ mass on the left side of the pelvis and behind the ovary. Computed tomography (CT) was not performed due to the patient's allergy to the contact medium. An atypical ultrasound appearance addressed the probable malignancy of the mass.

The patient underwent surgery. After opening the abdominal cavity, a free-fluid sample was taken, and the cytological result tested positive for malignant cells. The pelvic mass was firmly anchored to the small intestine, colon, sigma and uterine body (fig. 1). After removing the adhesions, double ureters were observed, which had been incorporated in the mass. The resection of the unilateral double ureters was necessary in order to remove the entire mass, after which the left ovary and tube were removed.

A histological examination showed a malignant retroperitoneal mass with clear nuclear polymorphism, whereas the right ovary and tube were unaffected. Anti-reflux terminoterminal ureteral anastomosis with two double-J stents was carried out (fig. 2). Total hysterectomy with preservation of the right adenexum and regional lymphadenectomy was performed. The postoperative recovery was uneventful, and after 7 days, the patient was discharged. The final histological results led to the diagnosis of a moderately differentiated retroperitoneal leiomyosarcoma. Radiotherapy completed the therapeutic management. After 1 year, the patient is healthy, and instrumental investigations are negative for recurrence. 
Leanza et al.: Retroperitoneal Sarcoma Involving Unilateral Double Ureter:

Management, Treatment and Psychological Implications

\section{Discussion}

RPS usually appears during the sixth decade of life. It typically exhibits few symptoms until it becomes large enough to compress or invade the surrounding structures. Patients may suffer from early satiety, obstructive gastrointestinal symptoms, pain or lower extremity swelling. The tumoral mass is usually discovered on physical examination when it is large and locally advanced. [1] The majority of such tumors have a low or medium grade of differentiation. Liposarcoma, leiomyosarcoma and malignant fibrous histiocytoma are the most common entities. Usually, leiomyosarcoma originates from the wall of the great vessels or from the female genital tract; it rarely originates from extragenital sites. In order to diagnose retroperitoneal leiomyosarcoma, a physical examination, laboratory studies and instrumental investigations must be carried out. However, for a definitive diagnosis, a histological result is mandatory. Laboratory studies should include the measurement of lactate dehydrogenase (an elevated level may be suggestive of lymphoma) and alphafetoprotein and beta-human chorionic gonodotropins (elevated concentrations are associated with germ cell tumors). An ultrasound, CT or magnetic resonance imaging scan of the entire abdomen and pelvis usually provide a satisfactory image of the primary mass [2]. Additionally recommended staging evaluations to detect metastatic disease depend on the histological grade of the lesion. Patients with known high-grade RPS should undergo a chest CT to detect lung metastases, whereas those with low-grade RPS can be satisfactorily evaluated by chest radiography [3]. According to the Memorial Sloan-Kettering Cancer Center guidelines, RPS is considered unresectable when vascular structures (e.g. aorta, vena cava and/or iliac vessels) are involved or when peritoneal implants, distant metastases and extensive disease are present. Likewise, the involvement of the mesenteric root and spinal cord are considered unresectable as well. Surgical removal with microscopically negative margins (R0) is the standard treatment for patients with localized, potentially resectable RPS. However, the large size and inability to remove the entire mass are reasons for RPS recurrence [4]. Radical surgical deletion often requires a resection of adjacent organs, as in our case. High recurrence rates after surgery have encouraged investigators to develop combined modality treatment approaches for patients with a high grade of invasion. However, due to the shortage of prospective randomized controlled trials, the usefulness of radiotherapy and chemotherapy is not well known. According to the severity of the lesion, therapy may be delivered preoperatively, intraoperatively or postoperatively. For our patient, surgery and postoperative prophylactic radiotherapy were performed to reduce the risk of recurrence.

QoL and psychological implications in women with urogynecological problems (e.g. urinary incontinence, sexually transmitted diseases) undergoing surgery have been examined in several research studies [5, 6]. In general, QoL for survivors of cancer appeared to be good, but a relatively large minority experienced depression, anxiety, distress or fear associated with recurrence or follow-up, particularly those who were younger, had more advanced disease with more severe physical symptoms and reduced social and economic resources. Based on the situation, the modified concept of the 'sick person' [7] is considered in a different way than the simple concept of 'person', causing a sort of dehumanization of the patient. As a result, these patients experience a lack of social functioning and a low QoL. Only a few psychosocial studies are available on quite young adults such as the case of the 45-year-old female patient reported here. Lifespan theories on human development suggest that the types of cancer-related negative consequences and the relevance recognized by such disruptions may vary according to the different stages of life. Young adults have futureoriented goals such as career planning, identity development, constructing stable relation- 
ships and families. Thus, they may experience greater disruptions in life as a result of their cancer. Prematurely confronting mortality can indeed produce significant distress in young individuals, as their goals in life might remain unfulfilled. Psychosocial factors for reducing emotional distress can be divided into internal (e.g. coping strategies, optimism, positive emotion) and external (e.g. environmental) factors. Among the internal factors, resilience has been reported to play a role in developing protective individual strategies in the adaptation to cancer. Resilience has been defined as the dynamic capacity to cope with adversities in life such as cancer. A number of psychological interventions, including cognitive behavioral therapy, acceptance and commitment therapy, and lifestyle modification may increase psychological resilience in the general population as well as in individuals with medical diseases. Among the external factors aimed at reducing emotional distress, social support has been broadly studied and is well known to help cancer patients cope with stress [8]. Nevertheless, some studies suggest that giving and receiving emotional support has positive effects on the emotional well-being of patients with a high competence in emotional communication, while the same social support can have a negative impact on the emotional well-being of those with a low competence in emotional communication. Thus, emotional dysfunctions (e.g. alexithymia) must be properly assessed and taken into consideration for the psychological treatment and management of cancer patients $[9,10]$. Finally, attachment styles may be important factors affecting QoL $[11,12]$.

The clinical case reported here is interesting for several reasons: (1) the rarity of RPS and the ambiguousness of diagnosis, (2) the ureteral implication, (3) the risk of kidney damage, (4) the psychological consequences, and (5) the combined treatment modalities.

Diagnosing sarcoma is not easy, and differentiating between myoma and sarcoma is sometimes challenging, especially when the sarcoma is not particularly large. Ureteral involvement may cause renal damage preoperatively, and the risk of either postoperative fistula or hydronephrosis must be considered, as in other dangerous obstetrical and gynecological diseases.

Psychological consequences may arise for many reasons: fear of malignant illness, hormonal privation and loss of the uterus. When a hysterectomy is carried out, a suitable support of the vaginal apex is compulsory to avoid apical compartment alterations and urinary symptoms following hysterectomy. Consequently, QoL could be altered.

Finally, combined treatment modalities and complete management of the disease can give the patient a better prognosis and an increased chance of survival. An unusual but successful case of soft tissue leiomyosarcoma involving unilateral double ureters such as the one presented herein has not been published previously in the literature.

\section{References}

1 Lewis JJ, Leung D, Woodruff JM, Brennan MF: Retroperitoneal soft- tissue sarcoma: analysis of 500 patients treated and followed at a single institution. Ann Surg 1998;228:355-365.

-2 Sanders TG, Parsons TW: Radiographic imaging of musculo skeletal neoplasia. Cancer Control 2001;8:221231.

3 Porter GA, Cantor SB, Ahmad SA, Lenert JT, Ballo MT, Hunt KK, Feig BW, Patel SR, Benjamin RS, Pollock RE, Pisters PWT: Cost-effectiveness of staging computed tomography of the chest in patients with T2 soft tissue sarcomas. Cancer 2002;94:197-204.

-4 Alektiar KM, Hu K, Anderson L, Brennan MF, Harrison LB: High-dose-rate intraoperative radiation therapy (HDR-IORT) for retroperitoneal sarcomas. Int J Radiat Oncol Biol Phys 2000;47:157-163.

5 Leanza V, Passanisi A, Leanza G: Urinary incontinence: quality of life and psychological aspects. UIJ 2013;27:e3.

6 Passanisi A, Leanza V, Leanza G: Impact of sexually transmitted diseases on quality of life: application of three validated measures. Giornale Italiano di Ostetricia e Ginecologia 2013;35:722-727. 


\section{Case Reports in Oncology}

\begin{tabular}{l|l}
\hline Case Rep Oncol 2014;7:301-305 & \\
\hline DOI: 10.1159/000363052 & $\begin{array}{l}\text { ○ 2014 S. Karger AG, Basel } \\
\text { www.karger.com/cro }\end{array}$ \\
\hline
\end{tabular}

Leanza et al.: Retroperitoneal Sarcoma Involving Unilateral Double Ureter: Management, Treatment and Psychological Implications

7 Hampton, JA, Passanisi, A, Jonsson, M: The modifier effect and property mutability. J Mem Lang 2011;64:233-248.

8 Burton NW, Pakenham K I, Brown WJ: Feasibility and effectiveness of psychosocial resilience training: a pilot study of the READY program. Psychol Health Med 2010;15:266-277.

-9 Caretti V, Porcelli P, Solano L, Schimmenti A, Bagby R M, Taylor GJ: Reliability and validity of the Toronto Structured Interview for Alexithymia in a mixed clinical and nonclinical sample from Italy. Psychiatry Res 2011;187:432-436.

10 Scimeca G, Bruno A, Pandolfo G, Micò U, Romeo V M, Abenavoli E, Schimmenti A, Zoccali R, Muscatello MR: Alexithymia, negative emotions, and sexual behavior in heterosexual university students from Italy. Arch Sex Behav 2013;42:117-127.

11 Schimmenti A, Passanisi A, Pace U, Manzella S, Di Carlo G, Caretti V: The relationship between attachment and psychopathy: a study with a sample of violent offenders. Curr Psychol 2014, Epub ahead of print.

12 Schimmenti A, Passanisi A, Gervasi AM, Manzella S, Famà FI: Insecure attachment attitudes in the onset of problematic internet use among late adolescents. Child Psychiatry Hum Dev 2013, Epub ahead of print.

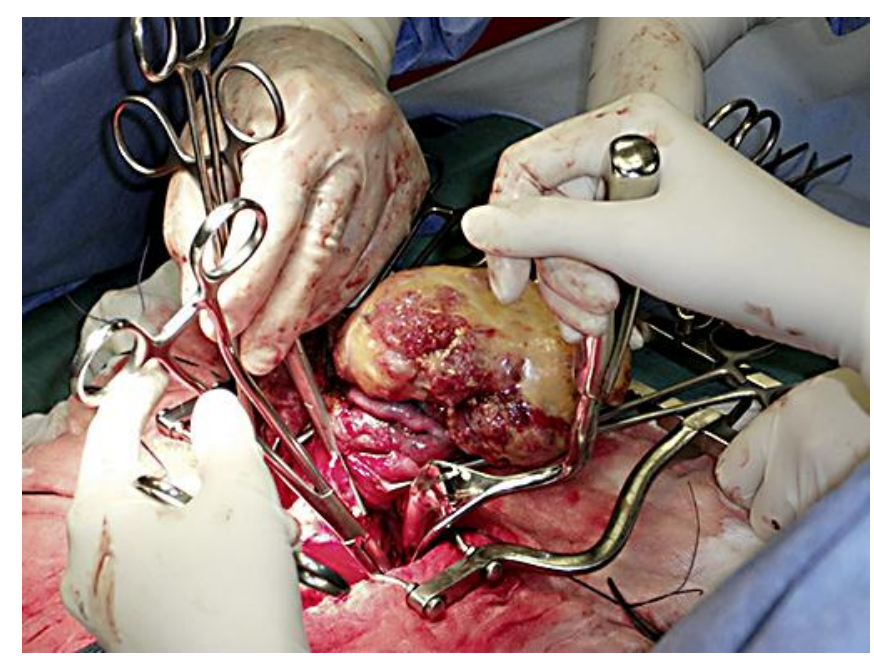

Fig. 1. Removal of the mass during surgery.

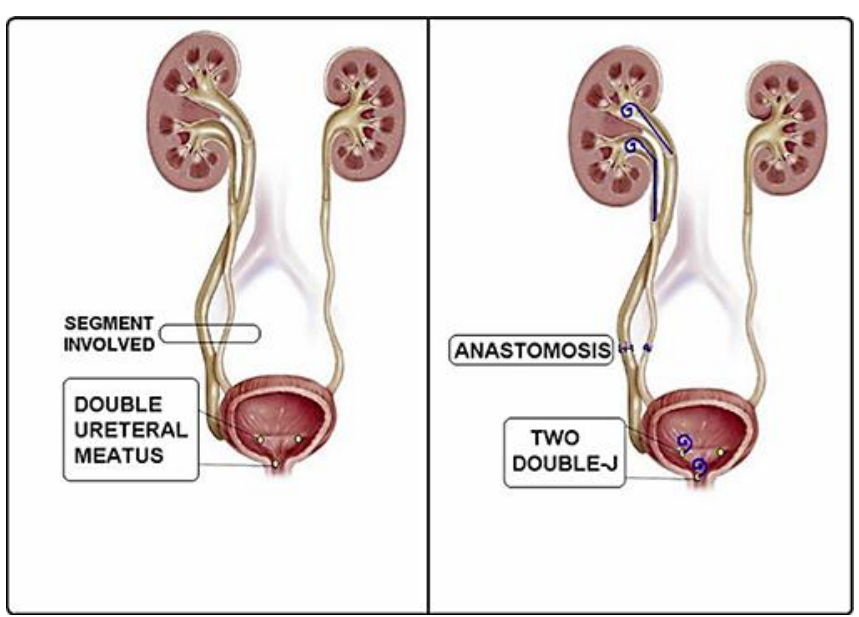

Fig. 2. Ureteral segment involved during mass removal (left side); ureteral anastomosis and two double-J insertions (right side). 